\title{
Mécanismes des hépatites médicamenteuses
}

La transformation des médicaments par le cytochrome $\mathbf{P}-\mathbf{4 5 0}$ aboutit parfois à la formation de métabolites toxiques. Malgré d'efficaces mécanismes de protection, ces métabolites peuvent être responsables d'hépatites.

\section{Dominique Pessayre} Directeur de Recherches à l'Inserm

\section{ADRESSE}

D. Pessayre : Inserm U 24, Hôpital Beaujon, 92118 Clichy cedex.

$m / s n^{\circ} 7, v o l .2$, septembre 86 es maladies iatrogènes, en particulier les hépatites, sont devenues une cause fréquente de morbidité. A l'hôpital Beaujon, les médicaments sont à l'origine de 10 \% des hépatites observées chez les sujets jeunes et de près de $50 \%$ chez les sujets âgés. Les hépatites médicamenteuses sont devenues, de fait, un problème majeur de santé publique. Le mécanisme de l'hépatite reste méconnu pour la plupart des médicaments. Certains progrès ont cependant été faits au cours de la dernière décennie dont l'un des plus importants a été de comprendre le rôle fréquent de métabolites instables [I-3].

Pour comprendre la fréquence de ce mécanisme, il convient de se souvenir du rôle du foie dans le métabolisme des médicaments.

\section{Rôle du foie}

Bien avant l'avènement des chimistes, les animaux et l'homme ont été exposés à une multitude de substances étrangères (ou xénobiotiques) ne faisant pas partie des constituants normaux des cellules vivantes. Ces xénobiotiques, présents dans l'eau de boisson, les plantes ou les ani-

Tableau I

MÉDICAMENTS HÉPATOTOXIQUES

CONNUS POUR ÊTRE TRANSFORMÉS EN MÉTABOLITES RÉACTIFS

Acétaminophène

Acide tiénilique

Alphaméthyldopa

Benoxaprofène

BCNU (Carmustine)

CCNU (Lomustine)

Chloramphénicol

Chloroforme

Chlorpromazine

Cyclophosphamide

Dantrolène

Enflurane
Erythromycine

Estrogènes naturels

Ethinylestradiol

Fluroxène

Halothane

Hycanthone

Imipramine

Iproniazide

Isaxonine

Isoniazide

Méthimazole

Méthoxyflurane
Métronidazole

Nitrofurantoïne

Noréthistérone

Phénacétine

Phénylbutazone

Phénytoïne

Propranolol

Propylthiouracile

Trichloroéthylène

Troléandomycine

Uréthane 


\section{RÉFÉRENCES}

I. Mitchell JR, Jollow DJ. Metabolic activation of drugs to toxic substances. Gastroenterology 1975; $68:$ 392-4 10.

2. Mazel P, Pessayre D. Significance of metabolite-mediated toxicities in the safety evaluation of drugs and chemicals. In: Mehlman GA, Shapiro RE, Blumenthal H, eds. Advances in Modern Toxicology. New Concepts in Sa fety Evaluation. Washington: Hemisphere Publishing Corporation, 1976; 1 : 307-43.

3. Pessayre D, Larrey D, Benhamou JP. Hépatites médicamenteuses. Sem Hop Paris 1985; 61 : 2049-7 I

4. Guengerich FP, Liebler DC. Enzymatic activation of chemicals to toxic metabolites. $C R C$ Crit Rev Toxicol 1985; 14 : 259-307.

5. Coles B. Effects of modifying structure on electrophilic reactions with biological nucleophiles. Drug Metab Rev 1984-85; 15 : 1 307-34.

6. Funck-Brentano C, Tinel M, Degott C, Lettéron $P$, Babany G, Pessayre D. Protective effect of 16,16 -dimethyl prostaglandin $\mathrm{E}_{2}$ on the hepatotoxicity of bromobenzene in mice. Biochem Pharmacol 1984; 33: 89-96.

7. Lettéron $P$, Fouin-Fortunet $H$, Tinel $M$ Danan G, Belghiti J, Pessayre D. Mechanism for isaxonine hepatitis. I. Metabolic activation by mouse and human cytochrome P-450. J Pharmacol Exp Ther 1984; 229: 845-50.

8. Fouin-Fortunet $H$, Lettéron $P$, Tinel $M$, Degott C, Fléjou JF, Pessayre D. Mechanism for isaxonine hepatitis. II. Protective role of glutathione and toxicological studies in mice 7 Pharmacol Exp Ther 1984; $229: 851-7$.

9. Breen K, Wandscheer JC, Peignoux M, Pessayre D. In situ formation of the acetaminophen metabolite covalently bound in kidney and lung. Supportive evidence provided by total hepatectomy. Biochem Pharmacol 1982; 31 : 1 I 5-6.

10. Babany G, Descatoire V, Corbic M, et al. Regulation of renal cytochrome P-450. Effects of two-thirds hepatectomy, cholestasis, biliary cirrhosis and post-necrotic cirrhosis on hepatic and renal microsomal enzymes. Biochem Pharmacol $1985 ; 34: 311-20$.

II. Pessayre D, Wandscheer JC, Descatoire V, Dolder A, Degott C, Benhamou JP. Cumulative effects of repeated doses of compounds transformed into reactive metabolites. Biochem Pharmacol 1980; 29: 1041-7.

12. Pessayre D, Cobert B, Descatoire V, et al. Hepatotoxicity of trichloroethylene-carbon tetrachloride mixtures in rats. A possible consequence of the potentiation by trichloroethylene of carbon tetrachloride-induced lipid peroxidation and liver maux consommés, devaient être éliminés. Le rein peut éliminer les substances solubles dans l'eau mais ne peut éliminer les substances solubles dans les graisses. Cette difficulté est contournée dans le foie où les xénobiotiques liposolubles sont d'abord oxydés par le cytochrome $\mathrm{P}-450$, puis conjugués en métabolites hydrosolubles qui vont pouvoir être éliminés dans les urines ou dans la bile (voir l'article de $P$. Beaune dans ce même numéro).

Grâce à la multiplicité des isoenzymes du cytochrome $\mathrm{P}-450$ d'une part, et d'autre part au fait que chaque isoenzyme peut accommoder de nombreux substrats, ce système est, dans son ensemble, capable d'oxyder une multitude de xénobiotiques différents. Un tel système représentait une solution économe permettant, avec un nombre limité d'enzymes, d'éliminer une infinité de xénobiotiques liposolubles différents. Ce système n'était cependant pas sans inconvénient.

\section{Formation de}

\section{métabolites réactifs}

Certains xénobiotiques sont oxydés par le cytochrome $\mathrm{P}-450$ en métabolites stables. D'autres xénobiotiques, incluant divers médicaments (tableau I, p. précédente), sont transformés en métabolites instables (figures $I$ et 2). Ces métabolites instables peuvent être formés par des mécanismes différents et ont des structures chimiques très diverses [4]. Ils ont en commun, cependant, la propriété d'attaquer spontanément les macromolécules hépatiques.

\section{Fixation covalente}

Véritables bombes moléculaires, les métabolites réactifs électrophiles (avides d'électrons) vont réagir spontanément et très rapidement avec divers nucléophiles tissulaires [5]. Le métabolite peut ainsi réagir avec le groupe $-\mathrm{SH}$ d'une cystéine, le soufre d'une méthionine, le $-\mathrm{NH}_{2}$ d'une lysine ou d'une arginine, ou le $-\mathrm{NH}-$ d'une histidine, et se fixer ainsi de façon covalente sur les protéines hépatiques (figure 3, p. 377). Un électrophile peut également réagir avec le $-\mathrm{NH}_{2}$, le $-\mathrm{N}=$ ou l'oxygène des bases puriques ou pyrimidiques, ou même avec l'oxygène du phosphate des acides nucléiques, et alkyler ainsi l'ADN ou l'ARN (figure 3). Un métabolite réactif donné peut se fixer sur de multiples protéines et/ou acides nucléiques différents. Il existe, cependant, un certain degré de sélectivité. Cette sélectivité fait intervenir en premier lieu la notion de "dureté " de l'électrophile ou du nucléophile [5]. Un électrophile ou un nucléophile est dit "dur" si son centre électrophile ou nucléophile est très polarisé. L'intérêt de cette classification est qu'un électrophile mou va réagir avec des nucléophiles mous tandis qu'un électrophile dur va réagir avec des nucléophiles durs. $\mathrm{Ci}$-dessus, les divers nucléophiles ont été cités par ordre de dureté croissante (du SH des protéines, mou, à l'oxygène des acides nucléiques, très dur). Il apparaît ainsi que les nucléophiles des protéines sont plus mous que ceux des acides nucléiques. Ainsi, un électrophile mou comme un aldéhyde réactif va réagir avec les protéines, mais pas avec les acides nucléiques [5]. Au contraire, un électrophile dur comme un époxyde polarisé, un ion nitrenium ou un ion carbonium va réagir avec les acides nucléiques [5]. On comprend ainsi que, selon sa dureté, un métabolite réactif puisse être à l'origine principalement de nécroses tissulaires ou principalement de cancers. Outre cette spécificité liée à la dureté de l'électrophile, d'autres facteurs structuraux interviennent, expliquant qu'un métabolite réactif va réagir plutôt avec telles ou telles protéines tandis qu'un autre métabolite alkylera plus volontiers d'autres protéines. Ce fait pourrait expliquer en partie que pour une même quantité de métabolite fixé sur les protéines hépatiques, l'hépatotoxicité de métabolites réactifs différents puisse être différente [6-8].

Le métabolite réactif étant très instable, il est peu capable de quitter son site de formation. Il réagit, de ce fait, principalement in situ à l'endroit même où il est formé [9]. Le cytochrome $\mathrm{P}-450$ est présent dans de nombreux organes. C'est dans le foie, cependant, que sa concentra- 
tion est la plus élevée [1o]. C'est donc le foie qui va principalement former ces métabolites réactifs et va être électivement soumis à leurs eff ets toxiques. La fixation du métabolite in situ explique donc le rôle important de ce mécanisme de toxicité dans le foie. Ce phénomène explique également la localisation de la nécrose. Le foie, en effet, est organisé en sous-unités appelées lobules. Le sang arrive dans la région périportale du lobule et termine son trajet dans la région centrolobulaire. C'est dans la région centrolobulaire que le cytochrome P-450 est le plus abondant. C'est donc principalement la région centrolobulaire qui va former les métabolites réactifs et qui sera électivement touchée par la nécrose qui peut en résulter.

Le métabolite fixé sur les protéines hépatiques ne peut quitter le foie que dans la mesure où ces protéines sont elles-mêmes soit sécrétées dans le plasma, soit scindées en leurs différents acides aminés. La demivie moyenne des protéines hépatiques est assez longue, de 24 à 48 heures chez le rat et plusieurs jours chez l'homme. Après administration d'une première dose du médicament, une partic du métabolite initialement fixé sur les protéines va persister dans le foie lorsque la deuxième dose est administrée. L'administration de cette deuxième dose va à nouveau accroître la quantité de métabolite fixé, et ainsi de suite jusqu'à l'obtention d'un plateau lorsque la quantité fixée après chaque dose devient égale à la quantité éliminée entre chaque dose par protéolyse et sécrétion [II]. Cette accumulation pourrait favoriser des processus de sensibilisation contre les complexes métabolite-macromolécules accumulés sur la membrane plasmique.

\section{Peroxydation lipidique}

Un deuxième type de lésion moléculaire peut être la survenue d'une peroxydation lipidique[12]. Effectivement, la fixation d'un radical libre $\left(R^{*}\right)$ sur un lipide insaturé, ou bien l'arrachement d'un atome d'hydrogène de ce lipide aboutit dans les deux cas à un radical lipidique (Lipide). Ce radical va fixer

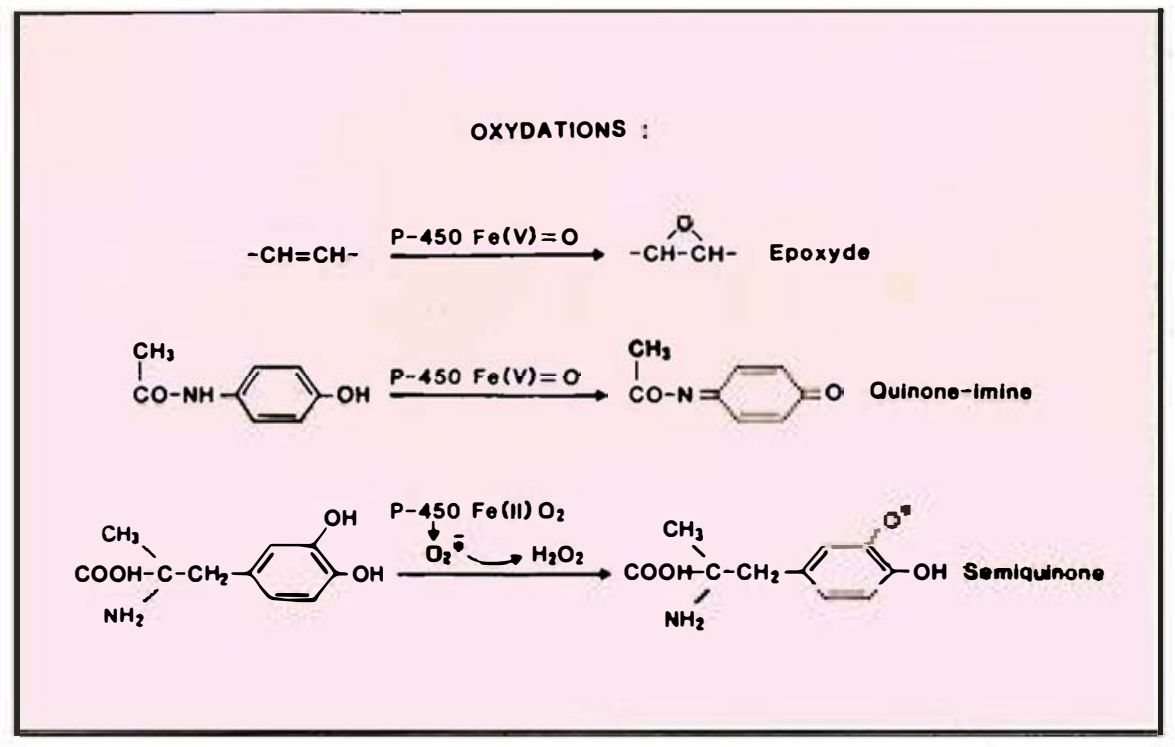

Figure 1. Formation de métabolites réactifs par oxydation. Les métabolites réactifs sont le plus souvent formés par oxydation. Celle-ci est en général réalisée par le complexe fer-oxygène actif du cytochrome $P$ - 450, probablement : $P-450 F e(V)=0$. La figure montre l'exemple de l'oxydation de la double liaison d'un arène ou d'un alcène en un époxyde, ainsi que l'oxydation de l'acétaminophène en une quinone-imine. Parfois, l'oxydation semble réalisée par un anion superoxyde libéré par le cytochrome P-450. On voit ici l'exemple de l'oxydation de l'a-méthyl-dopa en un radical semiquinone.

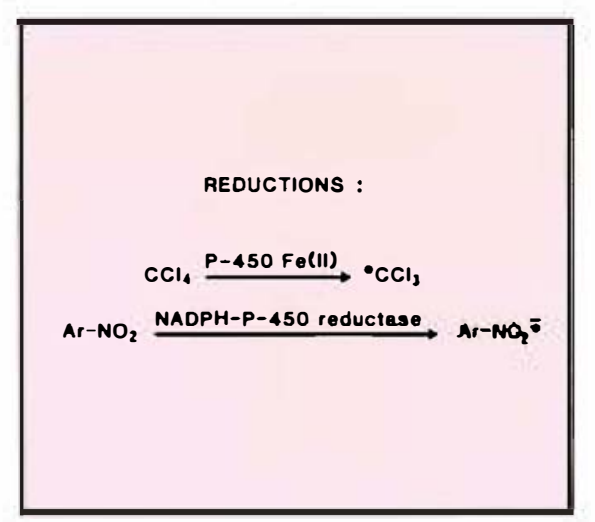

Figure 2. Formation de métabolites réactifs par réduction. Certains métabolites réactifs peuvent être formés par réduction. La forme réduite du cytochrome P-450 [P-450Fe (II)], se comporte comme un réducteur et peut déshalogéner certains haloalcanes. On voit ici la transformation du tétrachlorure de carbone en un radical trichlorométhyle. La NADPH-P-450 réductase peut directement réduire certains médicaments. C'est le cas de certains nitroarènes (comme le métronidazole ou la nitrofurantoine) qui sont transformés en un radical nitro. 
l'oxygène pour former un radical peroxy (Lipide-OO'). Ce dernier va réagir à nouveau avec une autre fonction insaturée lipidique pour former un hydroperoxyde et un nouveau radical lipidique :

$$
\begin{aligned}
& \text { Lipide }_{1}-\mathrm{OO}^{\circ}+\text { Lipide }_{2}-\mathrm{H} \\
& \rightarrow \text { Lipide }_{1}-\mathrm{OOH}+\text { Lipide }_{2}
\end{aligned}
$$

La peroxydation peut ainsi se propager d'une chaîne lipidique à l'autre, ou le long d'une même chaîne sur un lipide polyinsaturé. Finalement, les molécules de lipides insaturés peuvent être oxydées en plusieurs endroits, et débitées en petits fragments (alcanes, malondialdéhyde, alcénals). Ces deux derniers aldéhydes sont eux-mêmes réactifs et peuvent se fixer sur les protéines. Ainsi le cytochrome $\mathrm{P}-450$, bien que représentant un moyen idéal pour se débarrasser des xénobiotiques liposolubles, introduisait un nouveau problème en formant d'assez nombreux métabolites réactifs capables d'endommager les constituants cellulaires. A l'évidence, un tel système n'aurait guère pu être utilisé sans le développement préalable ou concomitant de divers mécanismes de protection.

\section{Destruction du cytochrome P-450}

17. Spielberg SP. Acetaminophen toxicity in lymphocytes heterozygous for glutathione synthetase deficiency. Can 7 Physiol Pharmacol 1985; $63: 468-71$.

18. Spielberg SP, Gordon GB, Blake DA, Goldstein DA, Herlong $\mathrm{HF}$. Predisposition to phenytoin hepatotoxicity assessed in vitro. $N$ Engl 7 Med $198 \mathrm{r} ; 305: 722-7$.

19. Larrey D, Lettéron $\mathrm{P}$, Foliot $\mathrm{A}$, et al. Efficts of pregnancy on the toxicity and metabolism of acetaminophen in mice. 7 Pharnacol Exp Ther 1986; $237: 283-91$.

20. Pessayre D, Dolder A, Artigou JY, et al. Effect of fasting on metabolitc-mediated hepatotoxicity in the rat. Gustroenterology 1979; 77 : 264-71.

21. Pessayre D, Bentata M, Degott C, et al. Isoniazid-rif ampin fulminant hepatitis. A possible consequence of the enhancement of isoniazid hepatotoxicity by enzyme induction. Gastroenterology' 1977; 72 : 284-9.

22. Pessayrc D, Mazel P. Induction and inhibition of hepatic drug metabolizing enzymes by rifampin. Biochem Pharmacol 1976; 25 : 943-9.

23. Pessayre D, Allemand H, Benoist C, Afifi F, François M, Benhamou JP. Effect of surgery under gencral anaesthesia on antipyrinc clearance. époxydes sont, de ce fait, réactifs. Il existe dans les microsomes et aussi le cytosol, diverses enzymes appelées époxydes hydrolases, qui hydratent les époxydes en dihydrodiols $(-\mathrm{CHOH}-\mathrm{CHOH}-)$ moins toxiques.

\section{Conjugaison au glutathion}

Un important mécanisme de protection est représenté par le glutathion [I]. Ce tripeptide, placé à forte concentration dans les hépatocytes (5 à Io $\mathrm{mM}$ ), va pouvoir se conjuguer, par son groupe-SH, avec de nombreux électrophiles réactifs et/ou réduire certains métabolites réactifs, protégeant ainsi les protéines hépatiques. La conjugaison au glutathion, cependant, consomme le glutathion dont les concentrations peuvent diminuer. Une forte diminution du glutathion a trois effets néfastes. Un premier effet est d'augmenter la fixation du métabolite réactif aux protéines : le métabolite réactif qui continue à être formé ne peut plus se conjuguer au glutathion et devient libre de se fixer massivement sur les protéines hépatiques. Une deuxième conséquence de la diminution du glutathion est d'altérer la fonction de nombreuses enzymes. En effet, un rôle important du glutathion est de maintenir les groupes-SH des protéines à l'état réduit. En l'absence de glutathion, le fonctionnement de nombreuses enzymes est, de ce fait, perturbé. C'est le cas, en particulier, des calcium translocases de la membrane plasmique dont le rôle est de rejeter les ions calcium hors de l'hépatocyte. Une troisième conséquence de la déplétion du glutathion est de permettre une peroxydation lipidique.

\section{Glutathion peroxydase, vitamine $E$}

Les hydroperoxydes lipidiques (R-OOH) et l'eau oxygénée $\left(\mathrm{H}_{2} \mathrm{O}_{2}\right)$ ont une certaine toxicité. En particulier ils risquent, en présence d'ions ferreux, de former respectivement un radical alcoxy ( $\left.\mathrm{RO}^{\circ}\right)$ ou un radical hydroxyle ( $\left.\mathrm{HO}^{*}\right)$, extrêmement réactifs. La glutathion peroxydase utilise le glutathion pour 


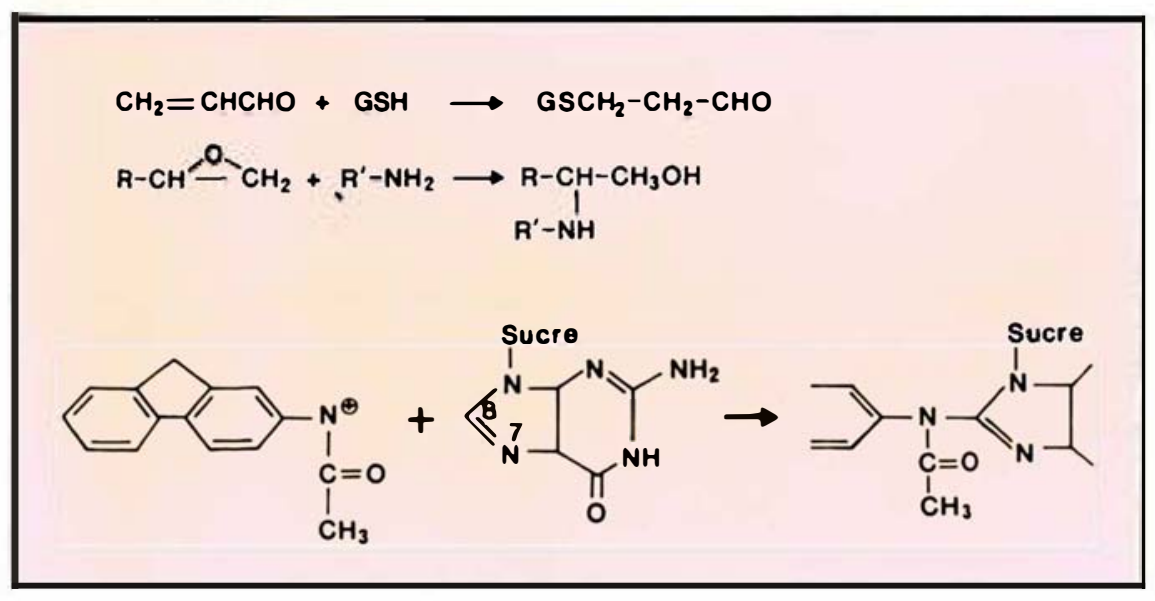

Figure 3. Fixation covalente sur les nucléophiles. On voit ici quelques exemples parmi les nombreuses réactions possibles entre un électrophile réactif et les nombreux nucléophiles tissulaires: addition de glutathion (GSH) sur la double liaison polarisée d'un aldéhyde insaturé en $\alpha$, $\beta$; fixation d'un époxyde réactif sur le $\varepsilon-\mathrm{NH}_{2}$ d'une lysine $\left(\mathrm{R}^{\prime}-\mathrm{NH}_{2}\right)$; réaction d'un ion nitrénium dérivant de l'acétylaminofluorène avec le $N_{7}$ d'une guanine, suivie de la migration de l'adduct sur le carbone adjacent $\left(C_{8}\right)$.

réduire les hydroperoxydes lipidiques ou l'eau oxygénée respectivement en alcools, et/ou en eau. Le glutathion oxydé formé lors de cette réaction est ensuite très rapidement réduit en glutathion (GSH) par la glutathion réductase. Outre la glutathion peroxydase, d'autres systèmes cytosoliques protéiques, encore incomplètement connus, sont capables de prévenir très efficacement la peroxydation lipidique en présence de glutathion réduit.

Un autre mécanisme de protection contre la peroxydation lipidique est la présence de vitamine $\mathrm{E}$ ( $\alpha$-tocophérol). Cette molécule a, d'une part, une longue chaîne aliphatique qui lui permet de s'inclure dans les membranes biologiques et, d'autre part, un noyau qui peut aisément donner un atome d'hydrogène. La vitamine $\mathrm{E}$ peut ainsi inactiver in situ les radicaux peroxy lipidiques, ainsi d'ailleurs que les formes particllement réduites de l'oxygène comme l'anion superoxyde $\left(\mathrm{O}_{\overline{2}}^{\overline{2}}\right)$, l'eau oxygénée $\left(\mathrm{H}_{2} \mathrm{O}_{2}\right)$ ou le radical hydroxyle $\left(\mathrm{HO}^{*}\right)$. Au cours de ces réactions, la vitamine $\mathrm{E}$ est consommée. De la vitamine $E$ native peut être cependant régénérée en présence d'acide ascorbique (qui est présent dans le réticulum endo- peutiques d'acétaminophène. Celuici entraîne donc également des hépatites idiosyncrasiques.

\section{Toxicité \\ idiosyncrasique}

Les médicaments ayant ce type de toxicité sont bien tolérés chez la grande majorité des sujets; chez quelques sujets cependant, l'ingestion de doses usuelles entraîne une hépatite toxique. Les raisons expliquant la sensibilité particulière de ces sujets restent mal connues. Elles pourraient être d'origine génétique, physiologique, nutritionnelle ou thérapcutique. Les différences génétiques pourraient porter en premier lieu sur les isoenzymes du cytochrome $\mathrm{P}-450$ (voir l'article de D. Larrey dans ce même numéro). En effet, l'activité d'un isoenzyme donné apparaît très variable d'un sujet à l'autre. Si le métabolite réactif est formé principalement par un isoenzyme donné, les variations de cet isoenzyme pourraient entraîner une grande variabilité dans la formation du métabolite. Les différences génétiques pourraient également porter sur les mécanismes d'inactivation. Certains sujets présentent un déficit partiel en glutathion synthétase et pourraient être plus sensibles à l'hépatotoxicité de l'acétaminophène et $d$ 'autres médicaments [iz]. D'autres sujets pourraient présenter un déficit en époxyde hydrolase et être plus sensibles à l'hépatotoxicité de la phénytoïne [18]. Ces hypothèses méritent cependant confirmation. Chez l'animal, des circonstances physiologiques telles que l'âge, le stress ou la grossesse[19] peuvent modifier le métabolisme et l'hépatotoxicité de certains médicaments. Le jeûne, total ou protéique, diminue le glutathion hépatique chez le rat et augmente considérablement l'hépatotoxicité de substances transformées en métabolites réactifs [20]. Chez l'homme, quelques cas d'hépatite ont été rapportés chez des sujets dénutris recevant de fortes doses thérapeutiques d'acétaminophène.

Un autre facteur capable d'augmenter la toxicité est la prise d'inducteurs enzymatiques. L'incidence des hépatites à l'isoniazide (un médica- 


\section{REFERENCES}

24. Pessayre D, de Saint Louvent P, Degott C Bernuau J, Rueff B, Benhamou JP. I proclozide fulminant hepatitis. Possible role of enzyme induction. Gastroenterology 1978; 75 : 492-6.

25. Pessayre D, Wandscheer JC, Cobert B, et al Additive effects of inducers and fasting on acetaminophen hepatotoxicity. Biochem Pharmacol 1980; 29 : 2219-23.

26. Allemand $\mathrm{H}$, Pessayre $\mathrm{D}$, Descatoire $\mathrm{V}$ Degott C, Feldmann G, Benhamou JP. Metabolic activation of trichloroethylene into a chemically reactive metabolite toxic to the liver. 7 Pharmacol Exp Ther 1978; 204: 71 -23

27. Pessayre D, Allemand $\mathrm{H}$, Wandscheer JC Descatoire V, Artigou JY, Benhamou JP. Inhibition, activation, destruction and induction of drug-metabolizing enzymes by trichloroethylene. Toxicol Appl Pharmacol 1979; $49: 355-63$.

28. Pessayre D, Lebrec D, Descatoire V, Peignoux M, Benhamou JP. Mechanism for reduced drug clearance in patients with cirrhosis Gastroenterology' 1978; $74: 566-7$ I.

29. Larrey D, Lebrec D, Bercoff E, Pessayre D Propranolol does not further decrease the clearance of antipyrine in patients with alcoholic cirrhosis. Clin Sci 1983; 65: 203-5.

30. Pessayre D, Larrey D, Funck-Brentano C, Benhamou JP. Drug interactions and hcpatitis produced by some macrolide antibiotics. Possible mechanisms. 7 Antimicrob Chemother 1986; I6 (suppl. A) : 181-94.

31. Pessayre D, Tinel M, Larrey D, Cobert B Funck-Brentano C. Inactivation of cytochrome $\mathrm{P}-450$ by a troleandomycin metabolite. Protective role of glutathione. 7 Pharmacol Exp Ther 1983; 224 : 685-91.

32. Vergani D, Mieli-Vergani G, Alberti A, et al Antibodies to the surface of halothane-altered rabbit hepatocytes in patients with severe halothane-associated hepatitis. $N$ Engl $7 \mathrm{Med}$ 1980; 303 : 66-71.

33. Neuberger J, Kenna JG, Nouri Aria K, Williams.R. Antibody mediated hepatocyte injury in methyl dopa induced hepatotoxicity. Gut 1985; 26: $1233-9$.

34. Danan G, Homberg JC, Bernuau J, RocheSicot J, Pessayre D. Hépatite à l'iproniazide. Intérêt diagnostique d'un nouvel anticorps antimitochondrial, l'anti-M $\mathbf{M}_{6}$. Gastroenterol Clin Biol I983; $7:$ 529-32.

35. Larrey D, Pessayre D, Duhamel G, et al. Prolonged cholestasis after ajmaline-induced acute hepatitis. 7 Hepatol 1986; 2 : 81-7.

36. Poupon R, Homberg JC, Abuaf N, Petit J, Bodin F Darnis F. Atteintes hćpatiques dues à l'acide tiénilique. Six observations avec présence d'anticorps anti-réticulum endoplasmique. Nouv ment transformé en un métabolite réactif) est augmentée par la prise concomitante de rifampicine [2I], un inducteur enzymatique [22], et peut-être aussi par la prise concomitante d'autres inducteurs, en particulier les médicaments utilisés pour la prémédication et l'anesthésie générale $[2 \mathrm{I}, 23]$. De même, les hépatites fulminantes à l'iproclozide semblaient déclenchées par l'adjonction, 7 à Io jours avant le début de l'ictère, de divers inducteurs enzymatiques [24]. Il convient de remarquer cependant que la prise d'un inducteur donné n'augmente pas toujours la toxicité d'un médicament donné. Il peut se faire, en effet, que les isoenzymes induits ne soient pas ceux qui forment le métabolite réactif du médicament, ou bien que l'inducteur induise également d'autres voies métaboliques de détoxication $[13,25]$. Fait intéressant, un médicament transformé en un métabolite réactif peut parfois induire lui-même sa propre transformation en un métabolite toxique $[26,27]$.

Plusieurs facteurs peuvent cumuler leurs effets. Chez le rat, les inducteurs et la déplétion du glutathion par le jeûne ont des effets additifs sur l'hépatotoxicité de l'acétaminophène [25]. Chez le rat toujours, l'administration d'alcool induit le cytochrome $\mathrm{P}-450$ et tend à diminuer le glutathion hépatique. Chez l'homme, des hépatonéphrites ont été observées chez des éthyliques chroniques recevant de fortes doses thérapeutiques d'acétaminophène.

La démonstration d'un mécanisme idiosyncrasique permet souvent diverses recommandations d'emploi. C'est ainsi que la reconnaissance du rôle toxique d'un métabolite de l'isoniazide a conduit aux recommandations suivantes : éviter les fortes doses d'isoniazide, éviter d'adjoindre à la rifampicine d'autres inducteurs enzymatiques et, en particulier, éviter de prescrire l'association isoniazide-rifampicine au décours immédiat d'une anesthésie générale; surveiller les transaminases, et, en cas d'élévation franche, interrompre l'administration d'isoniazide [2I] et non celle de rifampicine, comme on le faisait auparavant. Il semble que ces recommandations aient fortement diminué la fréquence des hépatites sévères. Une recommandation habituelle est d'éviter l'administration de médicaments hépatotoxiques chez les sujets cirrhotiques. Cette recommandation ne repose pas sur des bases scientifiques. La cirrhose diminue le métabolisme hépatique des médicaments [28] et pourrait ainsi diminuer la formation de métabolites réactifs [29]. Des études expérimentales et épidémiologiques devraient être entreprises pour clarifier cette question.

\section{Allergie}

Administré à doses thérapcutiques, le médicament entraîne chez de très rares sujets une hépatite ayant les caractères d'un phénomène allergique. Un tel mécanisme semble responsable des hépatites entraînées par de nombreux médicaments. C'est le cas, par exemple, des ictères à l'estolate d'érythromycine. Ces ictères sont rares; ils s'accompagnent fréquemment de manifestations allergiques (fièvre, hyperéosinophilie sanguine et, parfois, éruption cutanée) et récidivent précocement lors d'une deuxième administration [30]. L'estolate d'érythromycine est transformé par le cytochrome $\mathrm{P}-450$ en un nitrosoalcane [30]. Ce nitrosoalcane peut former un complexe inactif avec le fer (II) du cytochrome $\mathrm{P}-450$, et entraîner ainsi diverses interactions médicamenteuses. Le nitrosoalcane (ou la nitrone qui le précède) peut aussi réagir avec le glutathion ou la cystéine [3 I] et pourrait de ce fait se fixer irréversiblement sur les protéines hépatiques [30]. Tous les macrolides ne forment pas un tel nitrosoalcane. Les dérivés de l'érythromycine, et la troléandomycine forment ce nitrosoalcane et peuvent entraîner des interactions médicamenteuses ou des hépatites [30]. Au contraire, la josamycine, la midécamycine et la spiramycine, ne forment pas (ou peu) ce nitrosoalcane et n'entraînent pas (ou peu) d'interactions médicamenteuses ou d'hépatites [30]. Ainsi, il semble exister une relation entre le métabolisme des macrolides et la survenue d'une hépatite allergique. Il est tentant de spéculer que le métabolite irréversiblement fixé sur les pro- 
téines peut jouer le rôle d'un haptène et entraîner, chez quelques sujets, une immunisation contre les complexes métabolite-protéines de la membrane plasmique. Un tel mécanisme est proposé pour les hépatites à l'halothane [32], à l'isaxonine $[7,8]$, à l' $\alpha$-méthyldopa [33], et à l'acide tiénilique. Dans le sérum de sujets atteints d'hépatite à l'halothane ou à l' $\alpha$-méthyldopa, on trouve des anticorps dirigés contre des constituants hépatocytaires modifiés par l'exposition à ces médicaments $[32,33]$.

Lorsque l'administration du médicament est interrompue, les complexes métabolite-macromolécules antigéniques disparaissent progressivement de la surface hépatocytaire et la maladie guérit habituellement. Dans de rares cas, cependant, la maladie continue à évoluer malgré l'arrêt du médicament. De telles évolutions prolongées peuvent se voir dans certains cas d'hépatite cytolytique à l'iproclozide [24] ou à l'iproniazide [34]. Elles peuvent également s'observer dans certains cas d'hépatite cholestatique à l'ajmaline [35] : à l'arrêt du traitement, l'ictère guérit mais, chez quclques sujets, il persiste une cholestase prolongée associée à une raréfaction des petites voies biliaires intrahépatiques. De telles constatations posent le problème de l'existence possible de phénomènes auto-immuns associés.

\section{Auto-immunité}

La différence est faible entre les constituants hépatiques normaux et ceux qui, modifiés par la fixation du métabolite, auraient hypothétiquement entrainé une immunisation. Il est, de ce fait, envisageable que, chez certains sujets, la réaction immunitaire puisse également toucher, par réaction croisée, des constituants normaux de l'hépatocyte et/ou des cellules des petites voies biliaires. Une telle autoimmunisation associée permettrait d'expliquer la persistance prolongée de la maladie, malgré l'arrêt du traitement, chez certains malades. Elle permettrait aussi d'expliquer l'apparition possible de certains auto-anticorps. C'est ainsi que l'hépatite à l'iproniazide s'accompagne d'un auto-anticorps antimitochondrial particulier, l'anticorps anti-M6 [34]. De la même façon, l'hépatite à l'acide tiénilique s'accompagne d'un auto-anticorps anti-microsomes de foie et de rein [36]. La libération dans la circulation d'antigènes modifiés par le métabolite pourrait peut-être expliquer l'apparition de ces autoanticorps. Ce domaine reste cependant très mal élucidé. Dans l'état actuel des connaissances, l'existence d'hépatites allergiques ou autoimmunes d'origine médicamenteuse, bien que fortement suspectée sur des arguments indirects, reste hypothétique.

\section{Autres mécanismes}

Dans d'autres cas, c'est le médicament lui-même qui est toxique. L'incidence de l'hépatite augmente avec la dose administrée. L'hépatite ne récidive pas lors de la réadministration ultérieure d'une faible dose du médicament. Par exemple, l'hépatotoxicité du maléate de perhexiline semble due au médicament lui-même (voir l'article de D. Larrey dans ce même numéro). D'autres médicaments sont transformés en métabolites stables toxiques. Par exemple, le fluoro-uracile est transformé en acide fluorodésoxyuridylique qui inhibe la thymidilate synthétase. Les caractères de l'hépatotoxicité dans ces cas sont voisins de ceux observés lorsque c'est le médicament lui-même qui est toxique.

Les quelques exemples précédents ne doivent pas masquer le fait que le mécanisme de l'hépatite reste inconnu pour la plupart des médicaments [3]. Pour beaucoup d'entre eux, incluant certains médicaments très fréquemment hépatotoxiques, aucune étude n'a été entreprise pour essayer de comprendre le mécanisme des hépatites. Pourtant, la connaissance de ce mécanisme permet souvent de proposer diverses précautions d'emploi. Au cours des dernières années, certains laboratoires pharmaceutiques ont contribué activement à l'étude du mécanisme de l'hépatotoxicité des médicaments qu'ils commercialisent. Il est probable que cette attitude se généralisera

\section{Summary}

Several hepatotoxic drugs are transf ormed into chemically reactive metabolites which may be detoxified to stable metabolites or may covalently bind to cellular macromolecules. A few of these drugs produce toxic hepatitis in some subjects. This apparently idiosyncratic reaction may depend on genetic factors (cytochrome $\quad \mathbf{P}-450$ isozyme constitution, epoxide hydrolase activity, glutathione synthetase activity) as well as physiologic (age, pregnancy), nutritional (fasting, protein deficiency) or therapeutic influences (microsomal enzyme induction). With several other drugs, hepatitis appears to be allergic in type, possible triggered by antigenic metabolite-macromolecule complexes.

\section{TIRÉS A PART \\ D. Pessayre : Inscrm U 24, Hôpital Beaujon,} 92 I 8 Clichy cedex. 